\title{
Self-medication and related health complaints among expatriate high school students in the United Arab Emirates
}

\author{
Syed I. SHEHNAZ, Nelofer KHAN, Jayadevan SREEDHARAN, Khaled J. ISSA, Mohamed ARIFULLA.
} Received (first version): 14-Aug-2013 Accepted: 3-Nov-2013

\begin{abstract}
${ }^{*}$
Background: Self-medication, often without adult guidance, has been reported to be a common practice during adolescence. Similar to other preventable health-risk behaviors initiated in early adolescence, it has become a cause for concern universally.

Objectives: This study examines the prevalence of self-medication with both prescribed and nonprescribed (OTC) medications, related health complaints, sources of drugs, and sources of drug recommendation, and gender differences related to self-medication among expatriate high school students in the United Arab Emirates (UAE). Methods: A cross-sectional survey was conducted among 324 expatriate students through a validated, self-administered questionnaire and data was analyzed using SPSS 19 version. Means and proportions were calculated and Pearson Chisquare test of significance was used to analyze association among variables.

Results: Majority of the participating students, almost equally distributed by gender, was aged 16 to 17 years. The period prevalence rate of selfmedication with prescribed and OTC medications were $89.2 \%$, which did not vary with age, gender, ethnicity or parents' educational level. The most common sources of drug and drug recommendation were community pharmacies and parents respectively. Headache and fever were the common self-medicated conditions and consequently, analgesics and antipyretics were most commonly used both in the previous two weeks and the previous year prior to the survey. A high prevalence of self-medication with antibiotics $(53 \%)$ and sedative/hypnotics $(27 \%)$ was also observed. A female excess emerged for certain health complaints and use of medicines except for the use of anti-allergic and herbal/homeopathic drugs.
\end{abstract}

\footnotetext{
"Syed llyas SHEHNAZ. MBBS, MD, Assistant Professor, Department of Pharmacology, Gulf Medical University. Ajman (United Arab Emirates). shehnazilyas@yahoo.com Nelofer KHAN. PhD. Associate Professor. Department of Biochemistry, Gulf Medical University. Ajman (United Arab Emirates)

Jayadevan SREEDHARAN. PhD. Assistant Director, Statistical support Facility and Professor of Biostatistics. CABRI, Gulf Medical University. Ajman (United Arab Emirates).

Khaled Jamal ISSA. MBBS. Gulf Medical University. Ajman (United Arab Emirates).

Mohamed ARIFULLA. PhD. Dean (Admissions and

Registers) and Head of Department of Pharmacology, Gulf Medical University. Ajman (United Arab Emirates).
}

Conclusion: This is the first study to explore selfmedication practices among high school students in UAE and provides baseline data critical in creating awareness about the risks and benefits of selfmedication. Health care providers, educators and parents should be actively involved in health education strategies for inculcating responsible selfmedication practices in the adolescent population of UAE.

Keywords: Self Medication; Adolescent; Prevalence; Patient Medication Knowledge; Patient Education as Topic; United Arab Emirates

\section{AUTOMEDICACIÓN Y PROBLEMAS DE SALUD RELACIONADOS ENTRE ESTUDIANTES DE SECUNDARIA EXPATRIADOS EN LOS EMIRATOS ÁRABES UNIDOS}

\section{RESUMEN}

Antecedentes: Se ha informado de la práctica común de la automedicación entre adolescentes, a menudo sin vigilancia de un adulto. Al igual que otras actitudes de riesgo iniciadas en la adolescencia temprana, esto es de preocupación universal.

Objetivos: Este estudio examina la prevalencia de automedicación, tanto con medicamentos de prescripción como de medicamentos sin receta (OTC), los problemas de salud relacionados, las fuentes de medicamentos y las fuentes de recomendaciones de los medicamentos y las diferencias de géneros relacionadas con la automedicación entre estudiantes de secundaria expatriados en los Emiratos Árabes Unidos (UAE). Métodos: Se realizó un estudio transversal entre 324 estudiantes expatriados a través de un cuestionario validado auto-administrado y los datos se analizaron en un SPSS versión 19. Se calcularon las medias y las proporciones se utilizó la prueba chi-cuadrado de significancia para analizar la asociación entre variables.

Resultados: La mayoría de los estudiantes participantes, distribuidos igualmente por géneros, tenia de 16 a 17 años. La prevalencia de automedicación con medicamentos de prescripción y OTC en el periodo fue del $89,2 \%$, y no varió con la edad, género, etnia o nivel educativo de los padres. Las fuentes de medicamentos y de consejo sobre medicamentos más comunes fueron las farmacias comunitarias y los padres, respectivamente. El dolor de cabeza y la fiebre fueron las situaciones más frecuentes $\mathrm{y}$, 
consecuentemente, los analgésicos y los antipiréticos fueron los medicamentos más usados, tanto en las dos semanas previas a la encuesta como en el año anterior. Se observó también una alta prevalencia de automedicación con antibióticos (53\%) y sedantes/hipnóticos (27\%). Apareció un exceso de mujeres en el uso de ciertos problema de salud y uso de medicamentos, con excepción del uso de antialérgicos y medicamentos

vegetales/homeopáticos.

Conclusión: Este es el primer estudio que explora las prácticas de automedicación entre estudiantes de escuela secundaria en UAE y proporciona una base crítica para aumentar el conocimiento de los riesgos y beneficios de la automedicación. Los profesionales de la salud, educadores y padres deberían envolverse activamente en las estrategias educativas para inculcar prácticas responsables de automedicación en la población adolescente de UAE.

Palabras clave: Automedicación; Adolescente; Prevalencia; Conocimiento de la Medicación por el Paciente; Educación del Paciente como Asunto; Emiratos Árabes Unidos

\section{INTRODUCTION}

Self-medication is defined as obtaining and consuming drugs without the advice of a physician to treat self-recognized illnesses or symptoms. ${ }^{1,2}$ Generally involving over-the-counter (OTC) medications but also associated with prescriptiononly medicines (POM) ${ }^{3,4}$, self-medication forms a part of the concept of self-care and may be guided by a community pharmacist. ${ }^{2}$ Pharmacists can advise patients on drug choice and the "responsible" use of approved OTC drugs. ${ }^{1,2}$

Adolescence is a key period in which an individual takes first steps towards self-care and selfmedication. ${ }^{3,5-10}$ The health care habits adopted during adolescence may be carried over into adulthood. Moreover, adolescents may indulge in autonomous health behaviors, use medicine irrationally without adult guidance $e^{6-8,11}$ and also misuse OTC and prescription drugs to get "high". ${ }^{12}$ Additionally, a familial practice of SM has been reported to encourage SM during adolescence. ${ }^{10}$ The United Arab Emirates (UAE) is a Middle Eastern country of the Gulf Cooperation Council (GCC). An average $53.43 \%$ of the population of GCC countries (total population: 45.9 million $^{13}$ ) consists of expatriates. ${ }^{14}$ In the UAE, the expatriates constitute about $83 \%$ of the total population ${ }^{15}$ and those who live with their family are educated, professional qualified and belong to the middle/ higher income group. ${ }^{15}$ These expatriates may have access to a wide range of OTC medication (or even POM) in their home countries in addition to the OTC medicines available in UAE community pharmacies. Moreover, differences in accessibility to the public healthcare system between the local Emirati and expatriate population may also influence selfmedication practices in the latter.
Though globalization and migration have become a common phenomenon, published data on selfmedication practices in this large population (expatriates) are lacking and studies on the use of medicines by adolescents' (whether local or expatriate) from the Middle Eastern countries are scarce. ${ }^{8}$ As no previous study on medicine use by adolescents in the UAE could be identified, we felt it was worthwhile to explore the prevalence and practices of self-medication in this population.

The aims of the study were to explore the prevalence of self-medication with both POM and OTC medications, the health complaints related to self-medication, sources of drug procurement and sources of drug recommendation and to investigate any gender differences related to self-medication among expatriate high school students of UAE.

\section{METHODS}

A cross-sectional survey was carried out among a convenience sample of expatriate high school students (grades 9 to 12) in four private schools in the UAE, after obtaining Institutional Ethics Committee approval. The minimum sample size, calculated by considering the prevalence of selfmedication as $75 \%$ (based on average of prevalence rates of previous studies) $)^{8,7}$ and marginal error as $5 \%$, was 300 . Taking into account a non-response rate of $10 \%$, the questionnaires were distributed to the 330 students present on the day of the survey. The response rate was $98.2 \%$ with 324 students returning the completed questionnaires.

Male and female students majoring in natural sciences, commerce and arts were considered to obtain a representative sample from all three streams of study. The four participating schools had nearly equal distribution of gender in the classes which was reflected in our study sample. The teachers were briefed about the study objectives and the instructions for filling the questionnaires were given to, who then conveyed the information to their students. Voluntary participation was stressed upon, confidentiality of data was assured and informed consent was obtained from the participating students.

\section{Study questionnaire \& validation procedure}

A structured questionnaire, with close-ended statements, was developed through a series of focus group discussions with subject experts (three pharmacologists, two clinical pharmacists, two pediatricians and one internal medicine specialist) and review of literature. , $^{3,11,16,17}$ The questionnaire was content-validated by the subject experts and ambiguities in the statements were removed after pilot testing.

Operational terms were defined in the questionnaire as follows: Self-medication: use of OTC or POM for self-treatment, without prior consultation with a doctor; Doctor: any person who was medically qualified to prescribe medications; Medication: Substance used for treatment or prevention or 


\begin{tabular}{|c|c|c|c|}
\hline & Characteristic & $\begin{array}{l}\text { Number of students } \\
\text { self-medicating (\%) }\end{array}$ & $\begin{array}{c}\text { Number of students } \\
\text { not self-medicating (\%) }\end{array}$ \\
\hline \multirow{7}{*}{$\begin{array}{l}\text { Age } \\
n=291\end{array}$} & 14 years $(n=14)$ & $12(85.7)$ & $2(14.3)$ \\
\hline & 15 years $(n=38)$ & $31(81.6)$ & $7(18.4)$ \\
\hline & 16 years $(n=100)$ & $94(94.0)$ & $6(6.0)$ \\
\hline & 17 years $(n=92)$ & $81(88.0)$ & $11(12.0)$ \\
\hline & 18 years $(n=38)$ & $36(94.7)$ & $2(5.3)$ \\
\hline & 19 years $(n=09)$ & $8(89.0)$ & $1(11.0)$ \\
\hline & Not reported $(n=33)$ & $27(81.8)$ & $6(18.2)$ \\
\hline \multirow{3}{*}{$\begin{array}{l}\text { Gender } \\
\mathrm{n}=309\end{array}$} & Male $(n=149)$ & $134(89.9)$ & $15(10.1)$ \\
\hline & Female $(n=160)$ & $142(88.8)$ & $18(11.3)$ \\
\hline & Not reported $(n=15)$ & $13(86.7)$ & $2(13.3)$ \\
\hline \multirow{4}{*}{$\begin{array}{l}\text { Ethnicity } \\
\mathrm{n}=267\end{array}$} & $\begin{array}{l}\text { Asians of Indian } \\
\text { subcontinent }(n=129)^{*}\end{array}$ & $113(87.6)$ & $16(12.4)$ \\
\hline & $\operatorname{Arabs}(n=130)^{\star *}$ & $116(89.2)$ & $14(10.8)$ \\
\hline & Caucasians $(\mathrm{n}=8)^{* * *}$ & $6(75.0)$ & $2(25.0)$ \\
\hline & Not reported(n=57) & $54(94.7)$ & $3(5.3)$ \\
\hline \multirow{3}{*}{$\begin{array}{ll}\text { Paternal educational } \\
\text { level } \\
n=311\end{array}$} & Incomplete high school(n=26) & $22(84.6)$ & $4(15.4)$ \\
\hline & High school $(\mathrm{n}=61)$ & $52(85.2)$ & $9(14.8)$ \\
\hline & College $(n=224)$ & $202(90.2)$ & $22(9.8)$ \\
\hline \multirow{3}{*}{$\begin{array}{l}\text { Maternal } \\
\text { level } \\
n=310\end{array}$} & $\begin{array}{l}\text { Incomplete high } \\
\text { school }(n=33)\end{array}$ & $30(90.9)$ & $3(9.1)$ \\
\hline & High school $(n=86)$ & $74(86.0)$ & $12(14.0)$ \\
\hline & College $(n=191)$ & $172(90.1)$ & $19(9.9)$ \\
\hline
\end{tabular}

diagnosis of disease including modern medications or those from other healthcare systems.

The study questionnaire consisted of the following sections:

1. Socio-demographic details

2. Prevalence of self-medication: ascertained through a Yes or No response to a statement inquiring whether the students were taking medication without the advice of a doctor.

3. Age of commencing self-medication

4. Sources of drug procurement

5. Person who recommended the self-medication

6. Health complaints experienced in the preceding year

7. Drugs used in self-medication during the preceding year and the previous two weeks

The reference to a recall period of one year had the possibility of under-reporting of medicine use and therefore, the use of medicines during the past two weeks was also obtained. A list of options was included for sections 4 to 7 , following a review of literature indicating the commonly reported issues among self-medicating adolescents. ${ }^{3,5,7-9,11,16,17}$ Health complaints such as flu symptoms, allergies and eye problems were also included due to regional relevance. In order to avoid misinterpretation, the drug groups were specified based on their primary effects (e.g.: pain killers; fever relieving medicines; sleeping pills; anti-allergy medicines) and technical terms were avoided. All of them (except for antibiotics and sedatives/hypnotics) were either OTC or medicines which can only be sold by pharmacist according to the UAE regulations. ${ }^{18}$ The listed drugs were further classified according to Anatomical-TherapeuticChemical (ATC) Classification ${ }^{19}$ in the results.

\section{Statistical analysis}

Data analysis was done using SPSS 19 version. Means and proportions were calculated and the Pearson Chi-square test of significance was used to identify association among the dependent variable (self-medication) and the independent variables (age, gender, ethnicity and educational level of parents). The Spearman rank correlation coefficient was used to report the correlation between the number of health complaints and number of medicines self-medicated.

The period prevalence of self-medication was determined as the percentage of users out of the total sample who acquired a medicine that was not prescribed (written) or recommended (orally) by a physician. Any student who had a physician parent was not considered to be practicing self-medication.

The data of students who were self-medicating were further analyzed for sections 3 to 7 of the questionnaire. Several questions had multiple responses and therefore the percentages did not add up to a total of $100 \%$. Since the participants did not respond to every statement in the questionnaire, there were differences in the value of $n$ (the total population of respondents) for individual questions.

\section{RESULTS}

The socio-demographic characteristics of the 324 participating students appear in Table 1. All the students were expatriates from multi-ethnic backgrounds and almost equally distributed by gender. The majority were residing in the UAE for more than three years, aged 16 to 17 years with parents having college degrees.

The period prevalence rate of self-medication in the past year among the participants was $89.2 \%$, which 


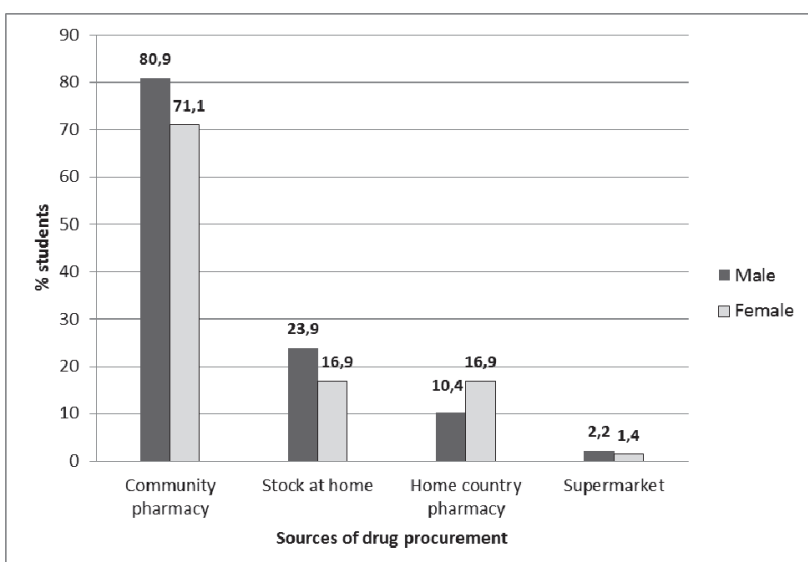

Figure 1. Sources of drug procurement (Males=134; Females=142)

did not vary with age, gender, ethnicity or educational level of the parents (Table 1). The majority of the respondents had started selfmedicating after the age of 13 years $(58.6 \%)$ but some also indicated $10-13$ years $(32.5 \%)$ and even $7-10$ years $(8.9 \%)$ as the age of commencing selfmedication.

The most common sources of the medicines among the self-medicating students $(n=289)$ were community pharmacies $(71.3 \%)$ and stocks kept at home $(20.4 \%)$ (Figure 1). Parents $(68.9 \%)$ and pharmacists $(46 \%)$ were the most common source of drug recommendation (Figure 2). There were no significant gender differences with regard to both these factors

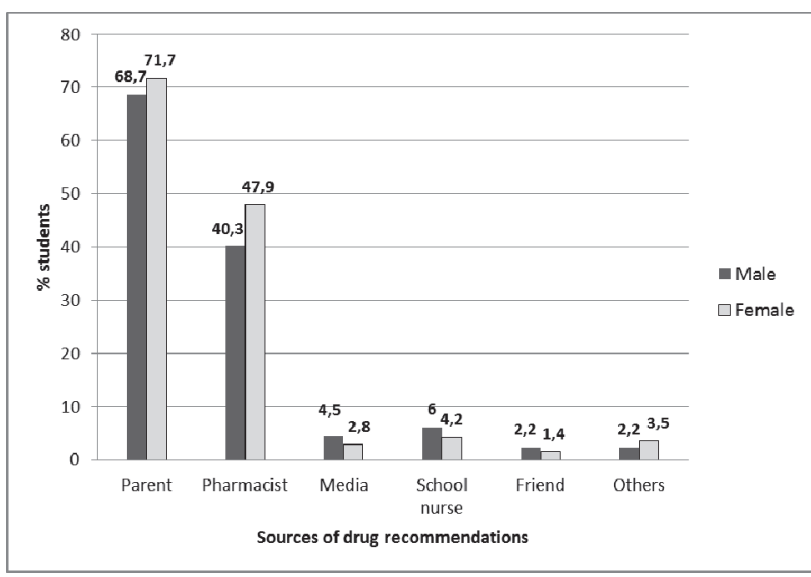

Figure 2. Sources of drug recommendation (Males=134; Females=142).

Headache, fever and flu symptoms were the common self- reported conditions in the previous year. About half of the students also reported allergies and sleep problems. Females suffered significantly more often with inability to sleep, skin, eye and ear diseases and constipation than the males (Table 2).

In concordance with the health complaints reported, antipyretics, analgesics and antibiotics were the medications used most commonly used during the past year. A significant female preponderance was observed for the use of vitamins/ nutritional supplements and sedative/hypnotics in the previous year (Table 3)

\begin{tabular}{|c|c|c|c|c|c|c|c|c|}
\hline \multirow{2}{*}{ Health complaints } & \multicolumn{6}{|c|}{ Number of students reporting frequency of health complaint in past one year $(n=289)$} & \multicolumn{2}{|c|}{$\begin{array}{l}\text { Gender distribution of } \\
\text { health complaint in past } \\
\text { one year\# }\end{array}$} \\
\hline & $\begin{array}{l}\text { At least } \\
\text { once a } \\
\text { year }(\%)\end{array}$ & $\begin{array}{c}\text { At least 2-3 } \\
\text { times a year } \\
(\%)\end{array}$ & $\begin{array}{c}\text { At least } \\
\text { Once a } \\
\text { month }(\%)\end{array}$ & $\begin{array}{c}\text { At least } \\
\text { Once a } \\
\text { week }(\%)\end{array}$ & $\begin{array}{l}\text { Almost } \\
\text { every day } \\
(\%)\end{array}$ & Total $(\%)$ & $\begin{array}{c}\text { Males }(\%) \\
n=134\end{array}$ & $\begin{array}{c}\text { Females }(\%) \\
n=142\end{array}$ \\
\hline Headache & $\begin{array}{c}45 \\
(15.6)\end{array}$ & $\begin{array}{c}83 \\
(28.7)\end{array}$ & $\begin{array}{c}52 \\
(18.0)\end{array}$ & $\begin{array}{c}58 \\
(20.1)\end{array}$ & $\begin{array}{c}19 \\
(6.6)\end{array}$ & $\begin{array}{c}257 \\
(88.9)\end{array}$ & $\begin{array}{c}117 \\
(87.3)\end{array}$ & $\begin{array}{c}129 \\
(90.8)\end{array}$ \\
\hline Fever & $\begin{array}{c}87 \\
(30.1)\end{array}$ & $\begin{array}{c}123 \\
(42.6)\end{array}$ & $\begin{array}{c}32 \\
(11.1)\end{array}$ & $\begin{array}{c}1 \\
(0.3)\end{array}$ & 0 & $\begin{array}{c}243 \\
(84.1)\end{array}$ & $\begin{array}{c}117 \\
(87.3)\end{array}$ & $\begin{array}{c}117 \\
(82.4)\end{array}$ \\
\hline $\begin{array}{l}\text { Flu/ cough / cold/ } \\
\text { Sore throat }\end{array}$ & $\begin{array}{c}48 \\
(16.6)\end{array}$ & $\begin{array}{c}127 \\
(43.9)\end{array}$ & $\begin{array}{c}42 \\
(14.5)\end{array}$ & $\begin{array}{c}16 \\
(5.5)\end{array}$ & $\begin{array}{c}2 \\
(0.7)\end{array}$ & $\begin{array}{c}235 \\
(81.3)\end{array}$ & $\begin{array}{c}109 \\
(81.3)\end{array}$ & $\begin{array}{c}116 \\
(81.7)\end{array}$ \\
\hline Stomach-ache & $\begin{array}{c}62 \\
(21.5)\end{array}$ & $\begin{array}{c}63 \\
(21.8)\end{array}$ & $\begin{array}{c}46 \\
(15.9)\end{array}$ & $\begin{array}{c}22 \\
(7.6)\end{array}$ & $\begin{array}{c}3 \\
(1.0)\end{array}$ & $\begin{array}{c}196 \\
(67.8)\end{array}$ & $\begin{array}{c}87 \\
(64.9)\end{array}$ & $\begin{array}{c}101 \\
(71.1)\end{array}$ \\
\hline $\begin{array}{l}\text { Pain in any other } \\
\text { part of body }\end{array}$ & $\begin{array}{c}58 \\
(20.1)\end{array}$ & $\begin{array}{c}50 \\
(17.3)\end{array}$ & $\begin{array}{c}35 \\
(12.1)\end{array}$ & $\begin{array}{c}25 \\
(8.7) \\
\end{array}$ & $\begin{array}{c}12 \\
(4.2) \\
\end{array}$ & $\begin{array}{c}180 \\
(62.3)\end{array}$ & $\begin{array}{c}84 \\
(62.7)\end{array}$ & $\begin{array}{c}88 \\
(62)\end{array}$ \\
\hline Allergy & $\begin{array}{c}81 \\
(28)\end{array}$ & $\begin{array}{c}45 \\
(15.6)\end{array}$ & $\begin{array}{c}22 \\
(7.6)\end{array}$ & $\begin{array}{c}6 \\
(2.1)\end{array}$ & $\begin{array}{c}11 \\
(3.8)\end{array}$ & $\begin{array}{c}165 \\
(57.1)\end{array}$ & $\begin{array}{l}75 \\
(56)\end{array}$ & $\begin{array}{c}81 \\
(57)\end{array}$ \\
\hline Inability to sleep & $\begin{array}{c}42 \\
(14.5) \\
\end{array}$ & $\begin{array}{c}23 \\
(8.0) \\
\end{array}$ & $\begin{array}{c}33 \\
(11.4) \\
\end{array}$ & $\begin{array}{l}26 \\
(9)\end{array}$ & $\begin{array}{l}23 \\
(8)\end{array}$ & $\begin{array}{c}147 \\
(50.9)\end{array}$ & $\begin{array}{c}57 \\
(42.5)^{\star}\end{array}$ & $\begin{array}{c}82 \\
(57.7)^{*}\end{array}$ \\
\hline Vomiting & $\begin{array}{c}92 \\
(31.8)\end{array}$ & $\begin{array}{c}43 \\
(14.9)\end{array}$ & $\begin{array}{c}8 \\
(2.8)\end{array}$ & $\begin{array}{c}2 \\
(0.7)\end{array}$ & 0 & $\begin{array}{c}145 \\
(50.2)\end{array}$ & $\begin{array}{c}60 \\
(44.8)\end{array}$ & $\begin{array}{c}79 \\
(55.6)\end{array}$ \\
\hline Skin problems & $\begin{array}{c}64 \\
(22.1)\end{array}$ & $\begin{array}{c}30 \\
(10.4)\end{array}$ & $\begin{array}{c}14 \\
(4.8)\end{array}$ & $\begin{array}{c}10 \\
(3.5)\end{array}$ & $\begin{array}{c}12 \\
(4.2)\end{array}$ & $\begin{array}{l}130 \\
(45)\end{array}$ & $\begin{array}{c}51 \\
(38.1)^{*}\end{array}$ & $\begin{array}{c}74 \\
(52.1)^{*}\end{array}$ \\
\hline Eye problems & $\begin{array}{c}68 \\
(23.5)\end{array}$ & $\begin{array}{c}30 \\
(10.4)\end{array}$ & $\begin{array}{c}12 \\
(4.2)\end{array}$ & $\begin{array}{c}7 \\
(2.4) \\
\end{array}$ & $\begin{array}{c}9 \\
(3.1) \\
\end{array}$ & $\begin{array}{c}126 \\
(43.6) \\
\end{array}$ & $\begin{array}{c}42 \\
(31.3)^{\star \star \star}\end{array}$ & $\begin{array}{c}78 \\
(54.9)^{\star \star \star}\end{array}$ \\
\hline Diarrhea & $\begin{array}{c}74 \\
(25.6)\end{array}$ & $\begin{array}{c}36 \\
(12.5)\end{array}$ & $\begin{array}{c}11 \\
(3.8)\end{array}$ & $\begin{array}{c}3 \\
(1)\end{array}$ & 0 & $\begin{array}{c}124 \\
(42.9)\end{array}$ & $\begin{array}{c}54 \\
(40.3)\end{array}$ & $\begin{array}{c}66 \\
(46.5)\end{array}$ \\
\hline Constipation & $\begin{array}{c}77 \\
(26.6)\end{array}$ & $\begin{array}{c}25 \\
(8.7)\end{array}$ & $\begin{array}{c}9 \\
(3.1)\end{array}$ & $\begin{array}{c}1 \\
(0.3)\end{array}$ & $\begin{array}{c}2 \\
(0.7)\end{array}$ & $\begin{array}{c}114 \\
(39.4)\end{array}$ & $\begin{array}{c}42 \\
(31.3)^{\star \star}\end{array}$ & $\begin{array}{c}68 \\
(47.9)^{\star *}\end{array}$ \\
\hline Ear problems & $\begin{array}{c}73 \\
(25.3)\end{array}$ & $\begin{array}{c}22 \\
(7.6)\end{array}$ & $\begin{array}{c}3 \\
(1.0)\end{array}$ & $\begin{array}{c}7 \\
(2.4)\end{array}$ & $\begin{array}{c}8 \\
(2.8)\end{array}$ & $\begin{array}{c}113 \\
(39.1)\end{array}$ & $\begin{array}{c}39 \\
(29.1)^{\star \star \star}\end{array}$ & $\begin{array}{c}71 \\
(50)^{\star \star \star}\end{array}$ \\
\hline $\begin{array}{l}\text { Menstrual } \\
\text { problems }\end{array}$ & $\begin{array}{c}36 \\
(12.5)\end{array}$ & $\begin{array}{c}25 \\
(8.7)\end{array}$ & $\begin{array}{c}36 \\
(12.5)\end{array}$ & 0 & 0 & $\begin{array}{c}97 \\
(33.6)\end{array}$ & NA & $\begin{array}{c}85 \\
(59.9)\end{array}$ \\
\hline
\end{tabular}




\begin{tabular}{|c|c|c|c|c|c|c|c|c|}
\hline \multirow{2}{*}{$\begin{array}{l}\text { Category of drugs } \\
\text { (ATC Code) }\end{array}$} & \multicolumn{6}{|c|}{ Number of students reporting frequency of self-medication in past one year $(n=289)$} & \multicolumn{2}{|c|}{$\begin{array}{c}\text { Gender distribution of } \\
\text { self-medication in past } \\
\text { one year\# }\end{array}$} \\
\hline & $\begin{array}{c}\text { At least } \\
\text { once a year } \\
(\%)\end{array}$ & $\begin{array}{c}\text { At least } 2-3 \\
\text { times a year } \\
(\%)\end{array}$ & $\begin{array}{c}\text { At least } \\
\text { once a } \\
\text { month (\%) }\end{array}$ & $\begin{array}{c}\text { At least } \\
\text { once a } \\
\text { week }(\%)\end{array}$ & $\begin{array}{c}\text { Almost } \\
\text { every day } \\
(\%)\end{array}$ & $\begin{array}{c}\text { Total } \\
(\%)\end{array}$ & $\begin{array}{c}\text { Males } \\
(\%) \\
n=134\end{array}$ & $\begin{array}{c}\text { Females } \\
(\%) \\
n=142\end{array}$ \\
\hline Antipyretics (N02B) & $\begin{array}{c}73 \\
(25.3)\end{array}$ & $\begin{array}{l}107 \\
(37)\end{array}$ & $\begin{array}{c}20 \\
(6.9) \\
\end{array}$ & $\begin{array}{c}2 \\
(0.7)\end{array}$ & $\begin{array}{c}1 \\
(0.3)\end{array}$ & $\begin{array}{c}203 \\
(70.2)\end{array}$ & $\begin{array}{c}90 \\
(67.2)\end{array}$ & $\begin{array}{c}106 \\
(74.6)\end{array}$ \\
\hline Analgesics (M01A/ N02B) & $\begin{array}{c}38 \\
(13.1) \\
\end{array}$ & $\begin{array}{c}96 \\
(33.2) \\
\end{array}$ & $\begin{array}{c}38 \\
(13.1)\end{array}$ & $\begin{array}{l}23 \\
(8)\end{array}$ & $\begin{array}{c}3 \\
(1) \\
\end{array}$ & $\begin{array}{c}198 \\
(68.5) \\
\end{array}$ & $\begin{array}{c}89 \\
(66.4) \\
\end{array}$ & $\begin{array}{c}101 \\
(71.1) \\
\end{array}$ \\
\hline Systemic Antibiotics (J01) & $\begin{array}{l}75 \\
(26) \\
\end{array}$ & $\begin{array}{c}65 \\
(22.5) \\
\end{array}$ & $\begin{array}{c}10 \\
(3.5)\end{array}$ & $\begin{array}{c}4 \\
(1.4) \\
\end{array}$ & - & $\begin{array}{c}154 \\
(53.2) \\
\end{array}$ & $\begin{array}{c}71 \\
(53) \\
\end{array}$ & $\begin{array}{c}82 \\
(57.7) \\
\end{array}$ \\
\hline $\begin{array}{l}\text { Vitamins/ nutritional } \\
\text { supplements (A11/B03) }\end{array}$ & $\begin{array}{c}55 \\
(19) \\
\end{array}$ & $\begin{array}{l}23 \\
(8) \\
\end{array}$ & $\begin{array}{c}12 \\
(4.2) \\
(4)\end{array}$ & $\begin{array}{c}13 \\
(4.5) \\
\end{array}$ & $\begin{array}{c}21 \\
(7.3)\end{array}$ & $\begin{array}{c}124 \\
(42.9) \\
\end{array}$ & $\begin{array}{c}49 \\
(36.6)^{*}\end{array}$ & $\begin{array}{c}71 \\
(50)^{*}\end{array}$ \\
\hline $\begin{array}{l}\text { Nasal decongestant } \\
\text { (R01A/R01B) }\end{array}$ & $\begin{array}{c}41 \\
(14.2)\end{array}$ & $\begin{array}{c}42 \\
(14.5)\end{array}$ & $\begin{array}{c}13 \\
(4.5) \\
\end{array}$ & $\begin{array}{c}7 \\
(2.4) \\
\end{array}$ & $\begin{array}{c}5 \\
(1.7) \\
\end{array}$ & $\begin{array}{c}108 \\
(37.4)\end{array}$ & $\begin{array}{c}48 \\
(35.8)\end{array}$ & $\begin{array}{c}56 \\
(39.4)\end{array}$ \\
\hline Anti-allergic Drugs (R06) & $\begin{array}{c}51 \\
(17.6)\end{array}$ & $\begin{array}{l}20 \\
(6.9)\end{array}$ & $\begin{array}{c}14 \\
(4.8)\end{array}$ & $\begin{array}{c}4 \\
(1.4) \\
\end{array}$ & $\begin{array}{c}5 \\
(1.7) \\
\end{array}$ & $\begin{array}{c}94 \\
(32.5) \\
\end{array}$ & $\begin{array}{c}38 \\
(28.4)\end{array}$ & $\begin{array}{c}52 \\
(36.6)\end{array}$ \\
\hline $\begin{array}{l}\text { Anti-emetic } \\
\text { Drugs(A04) }\end{array}$ & $\begin{array}{c}56 \\
(19.4) \\
\end{array}$ & $\begin{array}{c}22 \\
(7.6)\end{array}$ & $\begin{array}{c}3 \\
(1.0) \\
\end{array}$ & $\begin{array}{c}1 \\
(0.3) \\
\end{array}$ & - & $\begin{array}{c}82 \\
(28.4) \\
\end{array}$ & $\begin{array}{c}34 \\
(25.4) \\
\end{array}$ & $\begin{array}{c}46 \\
(32.4) \\
\end{array}$ \\
\hline $\begin{array}{l}\text { Sedatives/ } \\
\text { Hypnotics (N05C) }\end{array}$ & $\begin{array}{c}35 \\
(12.1) \\
\end{array}$ & $\begin{array}{c}17 \\
(5.9) \\
\end{array}$ & $\begin{array}{c}8 \\
(2.8) \\
\end{array}$ & $\begin{array}{c}12 \\
(4.2) \\
\end{array}$ & $\begin{array}{c}6 \\
(2.1) \\
\end{array}$ & $\begin{array}{c}78 \\
(27) \\
\end{array}$ & $\begin{array}{c}27 \\
(20.1)^{\star \star}\end{array}$ & $\begin{array}{c}48 \\
(33.8)^{\star \star}\end{array}$ \\
\hline Herbal/ Homeopathic & $\begin{array}{c}41 \\
(14.2)\end{array}$ & $\begin{array}{c}27 \\
(9.3)\end{array}$ & $\begin{array}{c}7 \\
(2.4)\end{array}$ & $\begin{array}{c}3 \\
(1.0)\end{array}$ & $\begin{array}{c}2 \\
(0.7)\end{array}$ & $\begin{array}{c}80 \\
(27.7)\end{array}$ & $\begin{array}{c}39 \\
(29.1)\end{array}$ & $\begin{array}{c}40 \\
(28.2)\end{array}$ \\
\hline $\begin{array}{l}\text { Anti-diarrheal } \\
\text { Drugs(A07) }\end{array}$ & $\begin{array}{c}56 \\
(19.4)\end{array}$ & $\begin{array}{c}13 \\
(4.5)\end{array}$ & $\begin{array}{c}2 \\
(0.7)\end{array}$ & $\begin{array}{c}2 \\
(0.7) \\
\end{array}$ & - & $\begin{array}{c}73 \\
(25.3) \\
\end{array}$ & $\begin{array}{c}32 \\
(23.9)\end{array}$ & $\begin{array}{c}39 \\
(27.5)\end{array}$ \\
\hline $\begin{array}{l}\text { Drugs for constipation } \\
\text { (A06) }\end{array}$ & $\begin{array}{c}44 \\
(15.2)\end{array}$ & $\begin{array}{c}18 \\
(6.2)\end{array}$ & $\begin{array}{c}4 \\
(1.4)\end{array}$ & $\begin{array}{c}1 \\
(0.3)\end{array}$ & $\begin{array}{c}3 \\
(1.0)\end{array}$ & $\begin{array}{c}70 \\
(24.2)\end{array}$ & $\begin{array}{c}28 \\
(20.9)\end{array}$ & $\begin{array}{c}40 \\
(28.2)\end{array}$ \\
\hline Others & $\begin{array}{c}21 \\
(7.3) \\
\end{array}$ & $\begin{array}{c}5 \\
(1.7) \\
\end{array}$ & $\begin{array}{c}4 \\
(1.4) \\
\end{array}$ & - & $\begin{array}{c}9 \\
(3.1) \\
\end{array}$ & $\begin{array}{c}39 \\
(13.5) \\
\end{array}$ & $\begin{array}{c}15 \\
(11.2) \\
\end{array}$ & $\begin{array}{c}23 \\
(16.2) \\
\end{array}$ \\
\hline
\end{tabular}

As with self-medication during the previous year, analgesics and antipyretics were most commonly used during the past two weeks too. Statistically significant gender differences were observed with the use of analgesics, sedative/hypnotics (higher in females) and anti-allergic, herbal/homeopathic medicines (higher in males) in the past two weeks (Table 4).

On sub-analysis of the data, the majority of selfmedicating students $(71.3 \%)$ reported experiencing at least one health problem in a month, with significant differences $(p<0.01)$ observed among males $(64.2 \%)$ and females $(78.2 \%)$. Similar significant gender differences $(p<0.001)$ were also observed in self-medication at least once in a month (Males: $38.8 \%$; Females: $58.4 \%$ ). A small fraction of students $(22.1 \%)$ reported at least one health complaint and $15.2 \%$ were partaking at least one drug on a daily basis. There were no gender differences in the health complaints suffered daily and daily use of drugs.

The students had experienced an average of 7.9 $(S D=4.2)$ health complaints in the past year. They had self-medicated with one to twelve drugs during the past year and with one to seven drugs during the past two weeks. The average number of drugs self-medicated during the past year and the past two weeks were $4.5(\mathrm{SD}=3.6)$ and $2(\mathrm{SD}=1.5)$ respectively. There was a statistically significant positive correlation between the number of health complaints and number of medicines self-medicated for both boys $(r=0.557 ; p<0.001)$ and girls $(r=0.648$; $p<0.001$ ).

\begin{tabular}{|c|c|c|c|}
\hline \multirow{2}{*}{$\begin{array}{l}\text { Category of drugs } \\
\text { (ATC Code) }\end{array}$} & \multirow{2}{*}{$\begin{array}{l}\text { Number of students self- } \\
\text { medicating at least once in } \\
\text { past two weeks }(\%) n=289\end{array}$} & \multicolumn{2}{|c|}{$\begin{array}{c}\text { Gender distribution of self-medication in } \\
\text { past two weeks\# }\end{array}$} \\
\hline & & $\begin{array}{c}\text { Males }(\%) \\
n=134\end{array}$ & $\begin{array}{c}\text { Females (\%) } \\
n=142\end{array}$ \\
\hline Analgesics (M01A/ N02B) & $149(51.6)$ & $61(45.5)^{\star}$ & $82(57.7)^{\star}$ \\
\hline Antipyretics (N02B) & $124(42.9)$ & $54(40.3)$ & $65(45.8)$ \\
\hline Nasal decongestants (R01A/R01B) & $63(21.8)$ & $29(21.6)$ & $27(19.0)$ \\
\hline Vitamins/Nutritional supplements(A11/ B03) & $54(18.7)$ & $25(18.7)$ & $26(18.3)$ \\
\hline Systemic Antibiotics(J01) & $33(11.4)$ & $15(11.2)$ & $18(12.7)$ \\
\hline Anti- allergic Drugs (R06) & $22(7.6)$ & $15(11.2)^{*}$ & $6(4.2)^{*}$ \\
\hline Anti-emetic Drugs (A04) & $26(9.0)$ & $10(7.5)$ & $13(9.2)$ \\
\hline Sedatives/Hypnotics(N05C) & $31(10.7)$ & $9(6.7)^{\star}$ & $21(14.8)^{*}$ \\
\hline Herbal/homeopathic Drugs & $25(8.7)$ & $17(12.7)^{\star \star}$ & $6(4.2)^{\star \star}$ \\
\hline Anti-diarrheal Drugs (A07) & $18(6.2)$ & $8(6.0)$ & $9(6.3)$ \\
\hline Drugs for constipation (A06) & $12(4.2)$ & $7(5.2)$ & $4(2.8)$ \\
\hline Others & $19(6.6)$ & $8(6.0)$ & $10(7.0)$ \\
\hline
\end{tabular}




\section{DISCUSSION}

Our study is the first that examined the selfmedication practices among the adolescent expatriate population in UAE. The high prevalence of self-medication, which illustrates autonomous health behavior in this population, is quite alarming. Recent research has also revealed that prescription and OTC drug abuse is an emerging trend ${ }^{12,20}$ and the use of medicines in adolescents is being regarded as part of a cluster of risk behaviors. ${ }^{21}$ Additionally, as most lifestyle habits are acquired during adolescence and as adolescents have inadequate knowledge about drugs ${ }^{7,22}$ this group is at high risk for irrational drug use.

Although other studies have used different recall periods, which limits comparison, the prevalence of self-medication in our sample is comparable to that reported in Kuwaiti $(92 \%)^{8}$ or Maltese $(90.3 \%)^{16}$ adolescents and higher than that observed in German $(57 \%)^{7}$ or Brazilian adolescents $\left(56.6 \%{ }^{3}\right.$, $\left.52.6 \%^{23}, \quad 50 \%{ }^{10}\right)$. Though self-medication is reported to increase with age among Danish ${ }^{5}$, Kuwaiti ${ }^{8}$, Saudi adolescents ${ }^{24}$; more common in German $^{7}$, Norwegian 9 , Brazilian ${ }^{10}$ females $^{6}$ and associated with maternal educational level in Brazil $^{10}$, no such associations were observed in our study.

Our data is concordant with literature ${ }^{16,25}$ that show pharmacies as the most common sources of medicines. Studies had also reported that adolescents had access to the home medicine cabinet $^{26}$ or obtained their medicines from their parents. ${ }^{5}$ Moreover, parents and pharmacists can probably influence adolescents on the appropriate use of drugs as the majority of our students reported to be self-medicating on their recommendation. Other studies have likewise identified mothers ${ }^{3,10}$ and pharmacists ${ }^{3,23}$ as being responsible for advising adolescents. Interestingly, only $5.2 \%$ of the cases of self-medication in our study were influenced by the media (including internet), analogous to that reported elsewhere. ${ }^{3}$

The high proportion of our students suffering from headache, fever and flu (as observed in other studies $3,7,11,17$ ) indicates the association of selfmedication with symptomatic treatment of these conditions. The high prevalence of self-reported allergies and sleeping problems is also notable as a lower prevalence of allergic rhinitis $(36 \%)^{27}$ and insomnia $\left(20 \%{ }^{11}, 15 \%{ }^{7}\right)$ had been reported elsewhere. Our data also confirms the adolescents' self-rated health as a predictor of medicine use because a positive correlation was found between the self-reported health complaints and medicine consumption for both genders.

The female excess observed for certain selfreported health complaints is also observed in other studies. ${ }^{28}$ Some researchers have explained these gender differences due to the differences in biology, psychological stresses, self-image, fixation with physical appearance, etc. ${ }^{29}$ The latter two reasons may contribute to the greater prevalence of skin and eye diseases reported by our female students. The higher proportion of females experiencing health complaints at least once a month $(78.2 \%)$ may also be attributed to the high prevalence of menstrual pain $(59.9 \%)$. The high monthly morbidity reported by females is also corroborated by their significantly higher use of analgesics in the preceding two weeks (Table 4).

As reported in other studies ${ }^{3,5,7-10}$, analgesics and antipyretics were the medicines most commonly used. Many of our students were also taking nutritional supplements $(43 \%)$, indicating their awareness of health-related issues. The use of antiallergics $(32.5 \%)$ is almost similar to that reported by Kuwaiti adolescents $(40 \%) .^{8}$ This high use of anti-allergics and nasal decongestants along with the high prevalence of self-reported allergies in our sample may suggest the need for further research on the regional prevalence of these disorders. The use of herbal/homeopathic medicine by a third of the sample indicates their acceptance of these alternative healthcare systems.

The one year prevalence rate of self-medication did not show any difference with regard to gender in our study. Nevertheless, on analyzing the practice of self-medication with respect to use of specific drug groups, a female excess was observed. Analogous to our study, female predominance of selfmedication has also been reported for pain (especially headache, stomach-ache) $)^{5,6,8,9}$ and sleeping difficulties ${ }^{5}$ (Tables 3 and 4). However, in contrast to the female excess observed with respect to vitamins/nutritional supplements in our study (Table 3 ), the use of vitamins was higher among male Kuwaiti adolescents. ${ }^{8}$ Moreover, unlike the significant male excess observed in the use of antiallergics over the past two weeks (Table 4), no such gender differences were reported in other studies. ${ }^{8,9}$

The high prevalence of self-medication with antibiotics $(54 \%)$ during the past year is alarming even though it is similar to that encountered among Indian adolescents $(58 \%){ }^{25}$ In contrast, Maltese $(19 \%)^{16}$, and Brazilian adolescents $(8.6 \%)^{3}$ seemed to be using antibiotics less often. The general population of UAE is also reported to be selfmedicating frequently with antibiotics acquired from community pharmacies without prescriptions ${ }^{30,31}$, though the OTC dispensing of antibiotic is illegal. ${ }^{18}$

Studies have indicated that immigrants were more likely to self-medicate ${ }^{32}$ and their prior experiences in countries with easy access to antibiotics influenced their acquisition of antibiotics without a prescription. ${ }^{33}$ The majority of expatriates residing in UAE come from developing countries where pharmacists often dispense antibiotics without prescriptions. 4,34 Consequently, as many of our expatriate adolescents appeared to be successful in obtaining antibiotics, it may be due to easy accessibility to stocks at home ${ }^{16,25,26}$ (bought from their own home countries or from community pharmacies in $U A E^{30,31}$ ) or "leftover" antibiotics from previous prescriptions. $^{26}$ Inappropriate selfmedication with antibiotics and the overall volume of antibiotic consumption in the community have serious implications in the development of antibiotic resistance. 
The use of sedatives/hypnotics by about one fourth of our sample is also noteworthy. Being a prescription drug in UAE, the sources for this may also be the same as those for antibiotics. Literature indicates a low prevalence of use of this drug $\left(6.4 \%^{6}, 15 \%^{7}\right)$. Furthermore, a female excess for sedatives/hypnotics was observed, which has also been reported by the Danish study ${ }^{5}$ but not confirmed by other studies. 6,9

The self-medication pattern observed in our respondents is similar to the practices adopted by adults. Analogous to our results, the use of analgesics/antipyretics and antibiotics is also commonly encountered among the adult population. ${ }^{4,35}$

The high overall use of medicines indicates that adolescents are taking responsibility for their own health but also exposes them to risks of misdiagnosis, inappropriate use, drug interactions and polypharmacy. Health care providers, educators and parents can play a vital role in making the transition to self-care successful in adolescents. ${ }^{36} \mathrm{~A}$ robust pharmacovigilance system is also advocated, with the community pharmacists providing advice to adolescents on responsible selfmedication under adult guidance. ${ }^{2}$ This extended role should be also taken into account in the education, training and practice of community pharmacists. Education and awareness campaigns can also be undertaken to promote the role of the pharmacist and enhance opportunities for adolescents communicating with pharmacists. More in-depth studies identifying and tackling the raison d'être of the use of antibiotics, sedatives, or hypnotics and education campaigns dealing with the effects of their inappropriate use directed at parents, adolescents and pharmacists can be advocated. Strict regulatory strategies should be enforced to limit the import of POM and their sale without prescription. OTC medicines may be dispensed to adolescents in limited numbers for the relief of mild illnesses.

We acknowledge the limitations of this standardized survey, particularly the self-reported nature of data and the likelihood of mutual influence between students .However, adolescents appear to report health risk behaviors and medicine use reliably over time. ${ }^{37}$ We are also aware that there could have been an overlap of use of Acetaminophen and NonSteroidal Anti-Inflammatory Drugs (NSAIDs) for the relief of pain and fever. Moreover, in view of the inclusion of schools with English as the medium of instruction (without representation from schools using Arabic or other languages), the small sample size and the non-probability sampling design, it may not be possible to generalize the results to the whole of UAE. Nevertheless, given the paucity of previous research, we consider that our study contributes to increasing the knowledge of medicine use in this population.

\section{CONCLUSIONS}

This is the first study to explore the self-medication practices among adolescent students in UAE and provides baseline data critical in creating awareness about the risks and benefits of self-medication. The prevalence of self-medication among the high school students in UAE was high, with no variation based on age, gender, ethnicity or parents' educational level. A high incidence of self-reported health complaints and a female preponderance of medicine use for certain health complaints were also discerned. As pharmacies were the most common source of self-medicated drugs and pharmacists were one of the common sources of drug recommendation, the community pharmacists have an important role as qualified public health educators in advising adolescents both on the choice of medicines and their safe and effective use. The extensive use of antibiotics and sedative/hypnotics indicates a necessity for educational health programs emphasizing the risks associated with their irrational use and strict enforcement of UAE Federal Laws regarding the dispensing of these drugs. Additional studies on local Emiratis adolescents and other expatriate ethnicities are directions for our future research. Health care providers, pharmacists, educators and parents should be actively involved in early health education strategies for establishing responsible use of medicines in this age group.

\section{ACKNOWLEDGEMENT}

The authors wish to thank Dr. Anoop K.Agarwal and Dr. Gamini Premadasa for their help in editing this article.

\section{CONFLICT OF INTEREST}

None.

Funding: This research received no specific grant from any funding agency in the public, commercial, or not-for-profit sectors.

\section{References}

1. World Health Organization. WHO guidelines for the regulatory assessment of medicinal products for use in selfmedication. Available at: http://apps.who.int/medicinedocs/en/d/Js2218e/1.html (Accessed 01/08/2013)

2. World Health Organization. The role of the pharmacist in self-care and self-medication. Report of the 4th WHO Consultative Group on the Role of the Pharmacist. The Hague, 1998. Available at: http://apps.who.int/iris/bitstream/10665/65860/1/WHO_DAP_98.13.pdf (Accessed 01/08/2013)

3. Pereira FS, Bucaretchi F, Stephan C, Cordeiro R. Self-medication in children and adolescents. J Pediatr (Rio J). 2007;83(5):453-458.

4. Awad A, Eltayeb I, Matowe L, Thalib L. Self-medication with antibiotics and antimalarials in the community of Khartoum State, Sudan. J Pharm Pharm Sci. 2005;8(2):326-331. 
5. Holstein BE, Holme Hansen E, Due P, Birna Almarsdóttir A. Self-reported medicine use among 11- to 15-year old girls and boys in Denmark 1988-1998. Scand J Public Health. 2003;31(5):334-341.

6. Hansen $\mathrm{EH}$, Holstein BE, Due $\mathrm{P}$, Currie $\mathrm{CE}$. International survey of self-reported medicine use among adolescents. Ann Pharmacother. 2003;37(3):361-366.

7. Stoelben S, Krappweis J, Rossler G, Kirch W. Adolescents' drug use and drug knowledge. Eur J Pediatr. 2000;159(8):608-614.

8. Abahussain E, Matowe LK, Nicholls PJ. Self-reported medication use among adolescents in Kuwait. Med Princ Pract. 2005;14(3):161-164.

9. Furu K, Skurtveit S, Rosvold EO. Self-reported medical drug use among 15-16 year-old adolescents in Norway. Tidsskr Nor Laegeforen. 2005;125(20):2759-2761.

10. da Silva $\mathrm{CH}$, Giugliani ER. Consumption of medicines among adolescent students: a concern. J Pediatr (Rio J). 2004;80(4):326-332.

11. Lau JT, Yu A, Cheung JC, Leung SS.Studies on common illnesses and medical care utilization patterns of adolescents in Hong Kong. J Adolesc Health. 2000;27(6):443-452.

12. Steinman KJ. High school students' misuse of over-the-counter drugs: a population-based study in an urban county. J Adolesc Health. 2006;38(4):445-447.

13. The cooperation council for the Arab states of the Gulf. Available at: http://www.gcc-sg.org/eng (Accessed 01/08/2013)

14. GCC Demographic Shift. Kuwait Financial Centre Markaz. Kuwait, 2012. Available at: http://www.markaz.com/DesktopModules/CRD/Attachments/DemographicsResearch-MarkazResearchJune\%202012.pdf (Accessed 01/08/2013)

15. National Bureau of Statistics. United Arab Emirates, 2012. Available at: http://www.uaestatistics.gov.ae (Accessed 01/08/2013)

16. Ellul RD, Cordina M, Buhagiar A, Fenech A, Mifsud J. Health complaints and use of medicines among adolescents in Malta. Pharm Pract (Granada). 2008;6(3):165-170.

17. Geissler PW, Nokes K, Prince RJ, Odhiambo RA, Aagaard-Hansen J, Ouma JH. Children and medicines: self-treatment of common illnesses among Luo schoolchildren in western Kenya. Soc Sci Med. 2000;50(12):1771-1783.

18. United Arab Emirates Ministry of Health. Registration and Drug Control Dept. Available at: http://www.haad.ae/HAAD/LinkClick.aspx?fileticket=8Yla2Sz8nDE\%3D\&tabid=207 (Accessed 01/08/2013)

19. WHO Collaborating Centre for Drug Statistic Methodology. ATC/DDD Index 2007. Available at: http://www.whocc.no/atcddd (Accessed 01/08/2013)

20. Apa-Hall P, Swartz R, McConnell R. The current state of teenage drug abuse: a trend toward prescription drugs. J School Nurs. 2008;24(Suppl):S1-S16.

21. Andersen $\mathrm{A}$, Holstein $\mathrm{BE}$, Hansen $\mathrm{EH}$. Is medicine use in adolescence risk behaviour? Cross-sectional survey of school-aged children from 11 to 15. J Adolesc Health. 2006;39(3):362-366.

22. Darmanin Ellul R, Cordina M, Buhagiar A, Fenech A, Mifsud J. Knowledge and sources of information about medicines among adolescents in Malta. Pharm Pract (Granada). 2008;6(4):178-186.

23. Moraes AC, Delaporte TR, Molena-Fernandes CA, Falcão MC. Factors associated with medicine use and self medication are different in adolescents. Clinics (Sao Paulo). 2011;66(7):1149-1155.

24. Nicholls PJ, Stevens RG, Albahsain NA. Medication used by Saudi girls. Saudi Pharm J. 2002;10(3):126-132.

25. Arul Prakasam KC, Senthil Kumar N. Assessment of health complaints and use of medicines among adolescents in Tamil Nadu. J Pharm Res. 2010;3(12):2799-2801.

26. Tourinho FS, Bucaretchi F, Stephan C, Cordeiro R. Home medicine chests and their relationship with self-medication in children and adolescents. J Pediatr (Rio J). 2008;84(5):416-422. doi:10.2223/JPED.1831

27. Alsowaidi S, Abdulle A, Shehab A, Zuberbier T, Bernsen R. Allergic rhinitis: prevalence and possible risk factors in a Gulf Arab population. Allergy. 2010;65(2):208-212. doi: 10.1111/j.1398-9995.2009.02123.x

28. Ellul RD, Cordina M, Buhagiar A, Fenech A, Mifsud J. An analysis of gender differences in self-reported health, use of medicines and access to information sources about medicines among adolescents. Int J Adolesc Med Health. 2009;21(4):591-600.

29. Torsheim T, Ravens-Sieberer U, Hetland J, Valimaa R, Danielson M, Overpeck M. Cross-national variation of gender differences in adolescent subjective health in Europe and North America. Soc Sci Med. 2006;62(4):815-827.

30. Abasaeed A, Vlcek J, Abuelkhair M, Kubena A. Self-medication with antibiotics by the community of Abu Dhabi Emirate, United Arab Emirates. J Infect Dev Ctries. 2009;3(7):491-497.

31. Dameh M, Green J, Norris P.Over-the-counter sales of antibiotics from community pharmacies in Abu Dhabi. Pharm World Sci. 2010;32(5):643-650. doi: 10.1007/s11096-010-9418-5

32. Carrasco-Garrido $P$, Jiménez-García R, Hernández Barrera V, López de Andrés A, Gil de Miguel A. Patterns of medication use in the immigrant population resident in Spain: associated factors. Pharmacoepidemiol Drug Saf. 2009;18(8):743-750. doi: 10.1002/pds.1776

33. Mainous AG 3rd, Diaz VA, Carnemolla M. Factors affecting Latino adults' use of antibiotics for self-medication. J Am Board Fam Med. 2008;21(2):128-134. doi: 10.3122/jabfm.2008.02.070149

34. Kotwani A, Wattal C, Joshi PC, Holloway K. Irrational use of antibiotics and role of the pharmacist: an insight from a qualitative study in New Delhi, India. J Clin Pharm Ther. 2012;37(3):308-312. doi: 10.1111/j.1365-2710.2011.01293.x

35. Yousef AM, Al-Bakri AG, Bustanji Y, Wazaify M. Self-medication patterns in Amman, Jordan. Pharm World Sci. 2008;30(1):24-30.

36. Geissler PW, Meinert L, Prince R, Nokes C, Aagaard-Hansen J, Jitta J, Ouma JH. What schoolchildren should be taught about medicines? Health Policy Plan. 2001;16(4):362-371.

37. Andersen A, Krølner R, Holstein BE, Due P, Hansen EH. Medicine use among 11- and 13-year olds: Agreement between parents' reports and children's self-reports. Ann Pharmacother. 2007;41(4):581-586. 